\title{
Effect of Mg-Based of Multi-Layered Structure on Hydrogen Desorption Properties
}

\author{
Ningning Zhou ${ }^{\mathrm{a}}$, Dongying Jü* \\ aPetrochemical Research Institute, Liaoning Petrochemical Vocational and Technology, No. 4, \\ District 2 Beijing Road, Jinzhou, Liaoning, 121001, China \\ ${ }^{\mathrm{b}}$ Department of Electronic Engineering, Saitama Institute of Technology, Fusaiji1690 Fukaya, \\ Saitama 369-0293, Japan \\ azhouningning_yes@163.com, bdyju@sit.ac.jp
}

Keywords: composite, sintering, absorption, desorption

\begin{abstract}
This study proposed the new method of preparing Mg-based composite by mixed powder $\mathrm{Ni}$ and $\mathrm{Ti}$ onto the surface of pure $\mathrm{Mg}$ ingot. The prepared method caused that hydrogen absorbing phase $\mathrm{Mg}_{2} \mathrm{Ni}$ and catalytic phase NiTi generated and distributed regularly. The pure $\mathrm{Mg}$ ingot as the center and the powder $\mathrm{Ni}$ and $\mathrm{Ti}$ as cladding material on the surface were formed and sintered, in which the temperature range of generated alloy phase $\mathrm{Mg}_{2} \mathrm{Ni}$ and NiTi was confirmed at first; according to the temperature range, the size of $\mathrm{Mg}_{2} \mathrm{Ni}$ and $\mathrm{NiTi}$ crystal grains at $650^{\circ} \mathrm{C}$ and $850^{\circ} \mathrm{C}$ were analyzed and compared, respectively; The size of $\mathrm{Mg}_{2} \mathrm{Ni}$ alloy phase at the surface and center was calculated by comparing the atomic radius of $\mathrm{Ni}$, Ti to confirm that $\mathrm{Mg}_{2} \mathrm{Ni}$ distributed on the surface due to the atom $\mathrm{Ti}$ replaced the atom $\mathrm{Ni}$ in $\mathrm{Mg}_{2} \mathrm{Ni}$ to generate the alloy phase $\mathrm{NiTi}$; the capacity of the hydrogen desorption reached $4 \mathrm{wt} \%$ within $5 \mathrm{~min}$. The disadvantages that easy to chalking and difficult to activate in the conventional method were avoided and achieved the application of the multilayer composite material in hydrogen storage field.
\end{abstract}

\section{Introduction}

In recent years, $\mathrm{Mg}$-based hydrogen storage material is very promising due to a large of hydrogen storage capacity, light weight, abundant resources and cheap price. Therefore, it is also regarded as the most promising hydrogen storage material. ${ }^{[1,2]}$ To adopt hydrogen storage material as a medium, the metal hydride are used in storage and transportation to resolve the long-term storage and safe transport issues. Therefore, many scholars have been considering the hydrogen storage material as an important direction of hydrogen energy. J.-M. Tarascon ${ }^{[3]}$ found that adding the rare earth elements can improve the formation mechanism of the alloy phases; effectively change the lattice spacing; significantly reduced hydrogen pressure in system and recycled temperature. The rare elements had obtained a wide application in the battery field, but the amount and the rate of hydrogen absorption and desorption still need to be further improved. X Tian ${ }^{[4]}$ prepared La-Mg-Ni based alloy material by adding other light elements into the rare elements. It is found that the alloy could achieve maximum discharge capacity and improve circulation stability by using a ball milling method, but the experimental method was easy to cause chalking and difficult to storage. B. Vigeholm and C.Stander ${ }^{[5]}$ had shown that the nano and amorphous materials could absorb and release hydrogen under low temperature. It is generally believed: the thermal stability of the nano and amorphous is poor and the crystal nuclear will grow in hydrogen storage cycle lead to the cycle life is poor and the dynamics is low, so hinder its development in practical application. In the present work, low price, abundant resources and light quality $\mathrm{Mg}$ based material was adopted. A new type prepared techniques included that synthesis-forming-sintering-sinter body achieved crystalline phase restructuring to generate the composite of clad $\mathrm{Mg}_{2} \mathrm{NiX}-\mathrm{NiTiX}$ (watchcase)- pure Mg (inner casing). Distinguishing conventional methods, the advantages from the powder and alloy were synthesized by the covered method that adding the powder onto the pure $\mathrm{Mg}$, so that the alloy phases $\mathrm{Mg}_{2} \mathrm{Ni}$ and NiTi generated continuously from the surface to the inner. The results showed that crystalline phase characteristic and organizational structure were improved and optimized, the capacity of the hydrogen desorption reached $4 \mathrm{wt} \%$ within $5 \mathrm{~min}$. 


\section{Experimental}

\subsection{Confirmation of sintering temperature range}

Pure $\mathrm{Mg}$ ingot was covered with the powder $\mathrm{Ni}$ and $\mathrm{Ti}$ at the molar ratio of $\mathrm{Ni}: \mathrm{Ti}: \mathrm{Mg}=\beta: \quad \gamma: \eta$ and was sintered at $10^{\circ} \mathrm{C} / \mathrm{min}$ to $1000^{\circ} \mathrm{C}$ by thermogravimetry-thermal difference(TG-DTA). As shown in Figure 1, the strong exothermic peak at $600^{\circ} \mathrm{C}$ indicated that the product formed due to the simple substances $\mathrm{Mg}$, Ni and Ti participated reaction. Therefore, it was confirmed that the alloy phases were generated. The purpose generated the alloy phases was to more visibly absorb and release hydrogen atoms and to form the composite of a coherent diffused layer. The weight loss of $4 \mathrm{mg}$ indicates that the volatility of $\mathrm{Mg}$ is comparatively obvious at a certain temperature. However, with exothermic peak, the reaction between the pure $\mathrm{Mg}$ and powder Ni caused the $\mathrm{Mg}_{2} \mathrm{Ni}$ phase tends to be more intense. Based on the material ratio, the obvious heat flow was caused due to the NiTi alloy phase played catalytic role may be formed while $\mathrm{Mg}_{2} \mathrm{Ni}$ phase formed near $600^{\circ} \mathrm{C}$. The results of TG-DTA showed that the alloy phase generated at about $600^{\circ} \mathrm{C}$. In order to confirm whether this experimental method can produce the hydrogen absorption phase and the catalytic phase, the high temperature X-ray diffraction was used to conduct the detection.

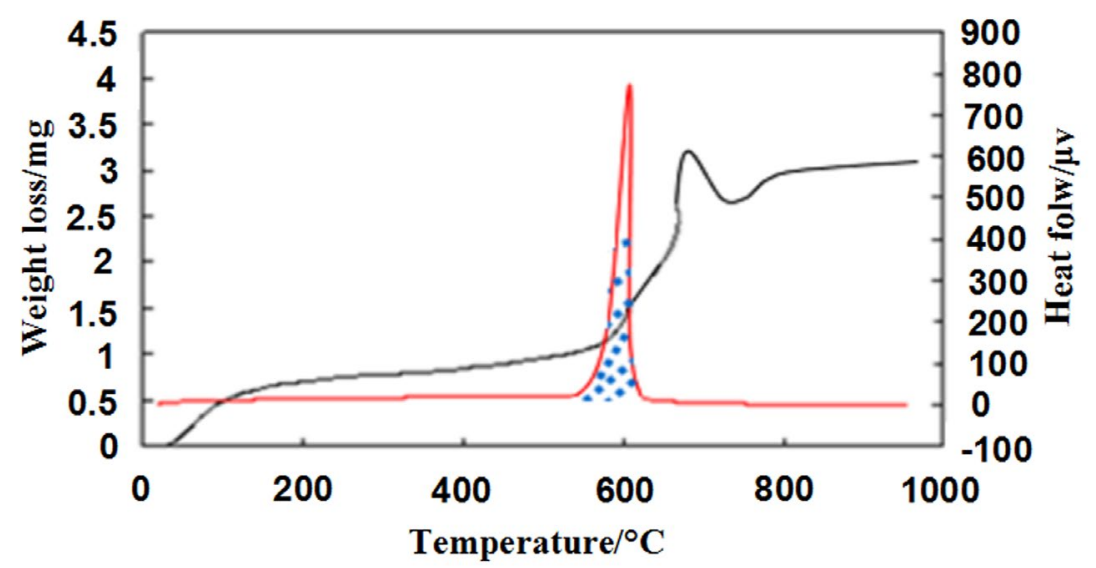

Fig. 1. TG-DTA analysis of simple substance $\mathrm{Mg}$, Ni and Ti to generated alloy phase.

\subsection{Refinement of grains under sintering temperature range}

As shown Figure 2, the alloy phases generated when the temperature increased to above $600^{\circ} \mathrm{C}$ according to the result of TG-DTA, the expansion of the lattice space between the two phases produced at the temperature of $650^{\circ} \mathrm{C}$ and $850^{\circ} \mathrm{C}$. There were relatively apparent the changes of the lattice space for $\mathrm{Mg}_{2} \mathrm{Ni}$, at the same time, there were no apparent the changes for the lattice space of NiTi. In accordance with the formula

(1)

$$
2 \mathrm{~d} \operatorname{Sin} \theta=n \lambda(\mathrm{n}=1)
$$

The difference between the interplanar spacings of $\mathrm{Mg}_{2} \mathrm{Ni}$ and $\mathrm{NiTi}$ under the different temperatures were obtained and represented as shown in (2) and (3)

$$
\begin{aligned}
& \mathrm{d}_{\mathrm{Mg}_{2} N i 850^{\circ} \mathrm{C}}-d_{M g_{2} N i 650^{\circ} \mathrm{C}}=\Delta d=0.021 \mathrm{~A} . \\
& \mathrm{d}_{N i T i 850^{\circ} \mathrm{C}}-d_{N i T i 650^{\circ} \mathrm{C}}=\Delta d=0.006 \mathrm{~A} .
\end{aligned}
$$

where $\mathrm{d}$ is the interplanar spacing, $\theta$ is the incident angle, $\lambda$ is the wavelength and $\mathrm{n}$ is the reflection coefficient. The direct contact of the powder $\mathrm{Ni}$, Ti with the $\mathrm{Mg}$ ingot at the above two temperatures can lead to the effective expansion of the surface area. Due to the higher purity and density of the pure $\mathrm{Mg}$ ingot, the reaction between $\mathrm{Mg}$ and Ni produced firstly to form the alloy phase $\mathrm{Mg}_{2} \mathrm{Ni}$ alloy. The trace addition of Ti powder is the major reason of the phenomenon of no apparent changes in the lattice 
space of the NiTi alloy. Although the pure Mg ingot was covered uniformly by the powder $\mathrm{Ti}$ and $\mathrm{Ni}$, there was still some weight loss with heating temperature (that is, very strong peak in Fig. 1). The results indicated that the grains of $\mathrm{Mg}_{2} \mathrm{Ni}$ coarsened with the heating temperature. The grain size can effectively expand the surface area of the composite material and can improve the activity of hydrogen absorption and adsorption, which had ultimately avoided the negative effects on the hydrogen absorption and desorption. Accordance to above results, the crystal particles were easy to grew coarse under the relative higher temperature $850^{\circ} \mathrm{C}$ (excessively coarse), so that the most suitable sintering temperature was $650^{\circ} \mathrm{C}-850^{\circ} \mathrm{C}$. The experimental temperature was confirmed about $750^{\circ} \mathrm{C}$ in the sintering process.

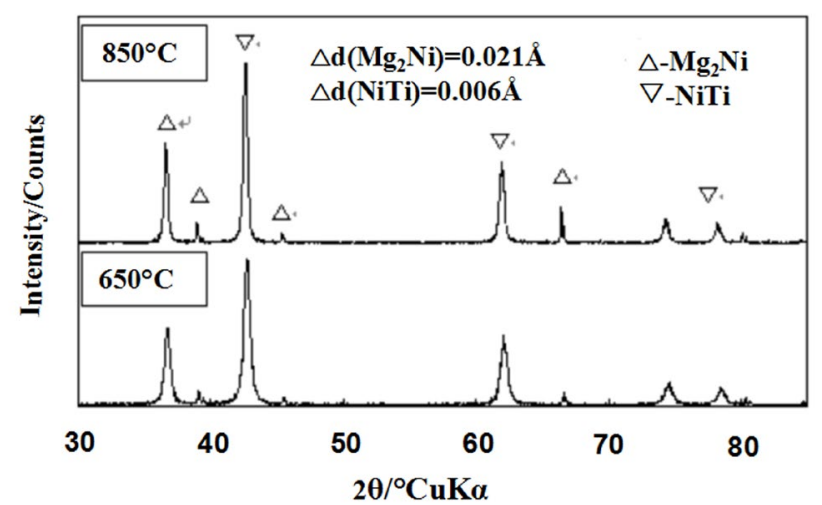

Fig. 2. XRD pattern of crystal particles refinement in sintered process.

\subsection{Characterization of crystal structure}

The experimental method improved the hydrogen absorption and desorption properties by way of synthesize the powder and the alloy was proposed. Meanwhile, The powder and the metal ingot, two types of materials that has distinctively different coefficients were used as the basic condition. Of these two materials, Ti was discussed as the major factor of changing crystal structure. As shown the Figure 3a, a special calculation method is used in this research to simulate the crystalline structure of $\mathrm{Mg}_{2} \mathrm{Ni}$ as the matrix. It is showed that the surface and the lattice space of the central position tend to be bigger.

$$
\Delta d_{M g_{2} N i}=0.024 \stackrel{\mathrm{O}}{\mathrm{A}} \text {. }
$$

It indicates that the direct contact of the powder with the $\mathrm{Mg}$ ingot in the central position promoted $\mathrm{Ti}$ participated in the reaction. The lattice spacing apparently changed between the surface and the central position, the major reasons were that the Ni atoms in the $\mathrm{Mg}_{2} \mathrm{Ni}$ alloy were displaced by $\mathrm{Ti}$, so that the expansion of crystal lattice and the semi-diameter of $\mathrm{Ni}$ atoms are relatively larger to form NiTi; accompanying the expansion of the volume.

$$
r_{T i}-r_{N i}=0.020 \stackrel{\mathrm{A}}{\mathrm{A}} \text {. }
$$

The difference ( $\Delta d_{M g_{2} N i}=0.024 \stackrel{\mathrm{O}}{\mathrm{A}}$ and $r_{T i}-r_{N i}=0.020 \stackrel{\mathrm{O}}{\mathrm{A}}$ ) was consistent, the result was confirmed once again that $\mathrm{Ti}$ entered the matrix of $\mathrm{Mg}_{2} \mathrm{Ni}$ and generated NiTi by way of displacement in Figure $3 b$. 


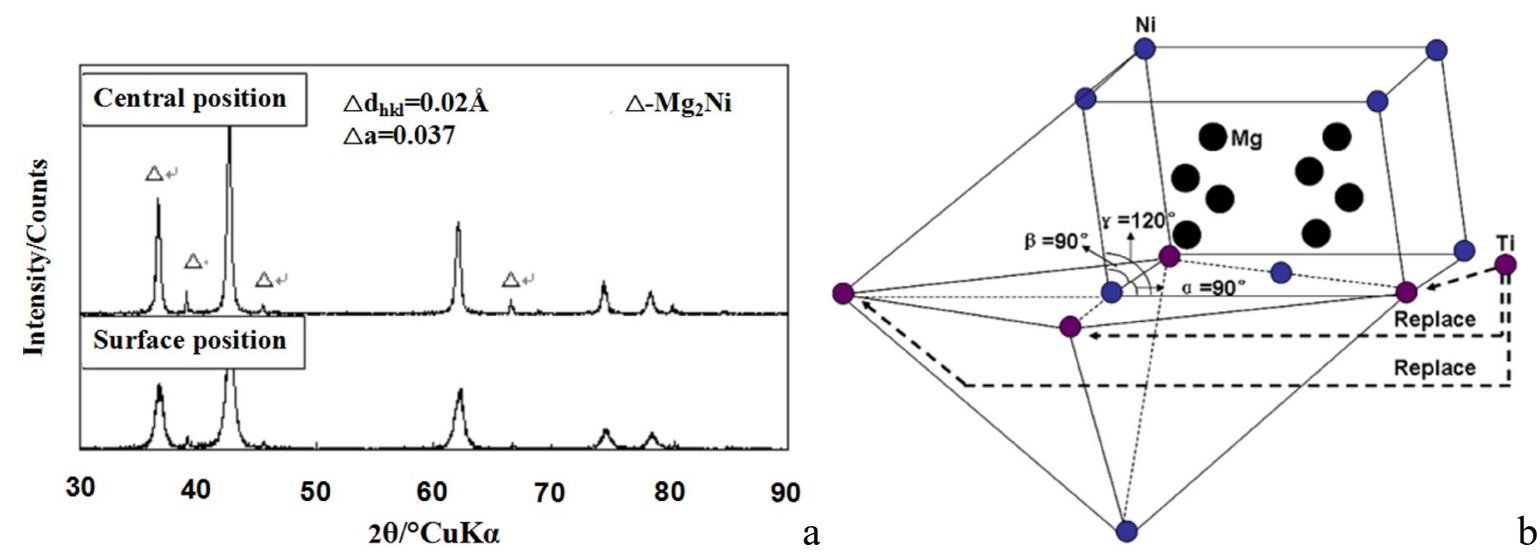

Fig. 3. Establishment of crystal structure model. a, XRD pattern of calculated lattice plane spacing by alloy phases distribution. $b$, Model of crystal structure.

\section{Results and Discussion}

\subsection{EDS elemental distribution}

As shown in Figure 4, the hexagonal structure of $\mathrm{Mg}_{2} \mathrm{Ni}(\mathrm{P} 6222)$ played hydrogen absorption role and distributed on the interface. the face-centered cubic structure of NiTi (Pm3m) phase formed in the inner of the composite and helped to promote hydrogen absorption/desorption as catalytic phase. $\mathrm{Mg}_{2} \mathrm{Ni}$ and NiTi phases generated in each diffused layer because the powder and the metal produced mutually diffusion and formed multi-layer structure. $\mathrm{Mg}_{2} \mathrm{Ni}$ distributed on the surface of the sintered body and a small amount distributed in the inner, which improved the hydrogen absorption capacity. But only $\mathrm{Mg}_{2} \mathrm{Ni}$ absorption phase did not improve the rate of hydrogen absorption/desorption. Therefore, NiTi catalytic phase as a medium could speed up the absorption/desorption rate and make $\mathrm{H}$ atoms diffused into the internal of the composite. NiTi phase not only stayed on the surface of the sintered body due to the mixed $\mathrm{Ni}$ and Ti powder reacted with pure $\mathrm{Mg}$ ingot in the sintering process, but also throughout the entire sintered body promoted the diffusion of $\mathrm{H}$ atom to the inner, so that improving the rate of hydrogen absorption/desorption. In which the lattice deformation formed due to the great difference between thermal expansion coefficients of the powder and pure Mg ingot, which provide a beneficial channel for $\mathrm{H}$ atoms diffusing into the internal of the sintered body.
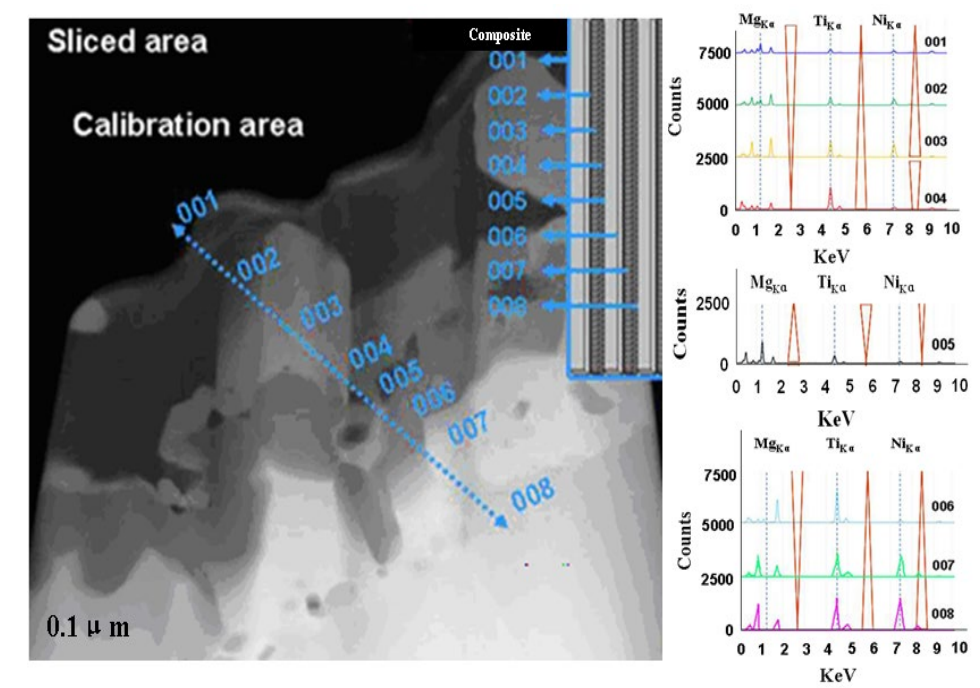

Fig. 4. TEM diffraction pattern and elemental distribution of $\mathrm{Mg}_{2} \mathrm{Ni}$ and NiTi.

\subsection{Temperature range of hydrogen absorption and desorption}

A special testing method was adopted to determine the temperature range of hydrogen absorption and adsorption. The basic condition is that the samples kept in the hydrogen environment for $48 \mathrm{~h}$ without 
any other conditions and then taken out. As shown in Figure 5, the endothermic peak occurred at about $340^{\circ} \mathrm{C}$. It showed that the weight loss of hydrogen released at this temperature was up to $4 \mathrm{wt} \%$ and the rate of hydrogen desorption was very fast about $5 \mathrm{~min}$.

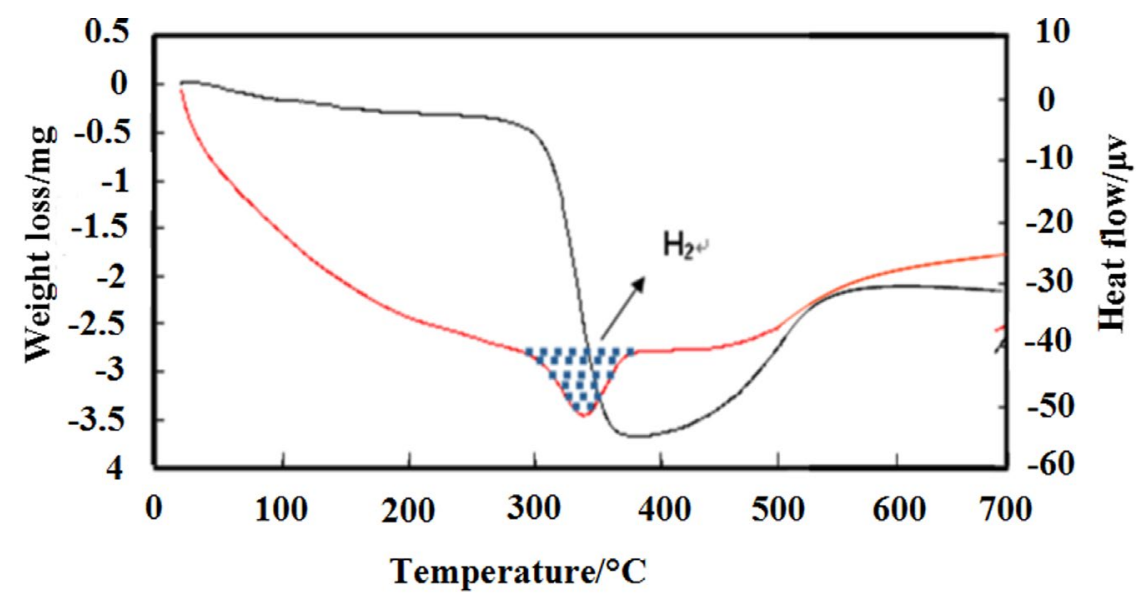

Fig. 5. TG-DTA analysis of hydrogen can be desorbed under a certain condition for confirming temperature range.

For produced negative factors from absorbing and desorbing hydrogen, many researchers from domestic and foreign had a large number of experiments to improve kinetic and thermodynamic properties. Alloy preparation had been attended. The amount of traditional $A_{5}, A B_{2}$ and $A B$ hydrogen storage materials are less than $2 \%$, the result limited the actual application of hydrogen storage materials. Therefore, high capacity hydrogen storage materials had been a focus in various fields. In the present, for a alloy, more attention was V-based solid solution hydrogen storage alloy; The theoretical amount of hydrogen storage of $\mathrm{VH}_{2}$ was up to $3.8 \%$, but the activated properties was poor lead to limit the widely application. The relative alloy had been further studied. The amount of hydrogen absorption and desorption after homogenization heat treatment could be raised to $2 \%$. It had been one of research hotspots. But the study on the affects from temperature and $\mathrm{H}_{2}$ pressure to hydrogen absorption and desorption is not sufficient. The study by using a new laminated cover method and changing element was conducted and was discussed to prepare the special structure sintered body. It had a large surface area can effectively conduct the cycle of hydrogen absorption and desorption; so that achieved the whole cycle was completed within very short time. In the process, the amount of hydrogen absorption and desorption was obviously improved, in which the amount of hydrogen desorption reached $4 \%$ within $5 \mathrm{~min}$.

\section{Conclusion}

The study synthesized advantages of the powder and the metal to optimize the structure of the crystal through adding trace element $\mathrm{Ti}$; the composite effectively overcame inconvenient from the powder on storage aspect and avoided negative factors of small surface area due to an increasing of the grain boundary between the crystal grains and the channels of $\mathrm{H}$ entered into the material increased. For the sintered body, the amount and the rate of hydrogen desorption was obviously improved and even superior to the effects from a powder by traditional method. The amount of hydrogen desorption reached $4 \%$ within $5 \mathrm{~min}$. 


\section{References}

[1] Darci L. Savickia, 1, Horácio A. Vielmoa and Arno Krenzingerb, Three-dimensional analysis and investigation of the thermal and hydrodynamic behaviors of cylindrical storage tanks, Renewable Energy, 2010, 10, 011.

[2] Zhao Y, Gao M X, Liu Y F, Huang L, Pan H G, The correlative effects of Al and Co on the structure and electrochemical properties of a La-Mg-Ni-based hydrogen storage electrode alloy. J. Alloys Compounds. 2010, 496, 454.

[3] Jean-Marie Tarascon, Nadir Recham, Hunting for Better Li-Based Electrode Materials via Low Temperature Inorganic Synthesis. Chem. Mater, 2010, 22, 724-739.

[4] Hai-Liang Chu, Shu-Jun Qiu, Effect of ball-milling time on the electrochemical properties of La-Mg-Ni-based hydrogen storage composite alloys. International Journal of Hydrogen Energy. 2007, 32, 4925-2932.

[5] B. Vigeholm, B. Larsen, Hydrogen sorption performance of pure magnesium during continued cycling. International Journal of Hydrogen Energy. 1883, 8, 809-817. 